\title{
Arthroscopy and HRUS Correlation in IDK
}

\author{
Dr. A. Sundeep Kund Reddy ${ }^{1}$, Dr. T. Rajani ${ }^{2}$ \\ I (MS(Ortho); DNB(Ortho); MCh(Ortho), Chief Orthopaedic Surgeon, Jai Hind Center For Ortho \& Radiology \\ / Hyderabad, India) \\ ${ }_{2}^{2}$ (DNB(Radio-Diagnosis), Chief Radiologist, Jai Hind Center For Ortho \& Radiology / Hyderabad, India)
}

\begin{abstract}
In a prospective study we invistigated internal derangement of knee (IDK) with High resolution ultrasound (HRUS). Aim was to determine effectiveness of ultrasound in diagnosing IDK and to compare with Arthroscopy. Ultrasound showed good sensitivity (91.3\%) and specificity (90.6\%) and the figures were comparable toarthroscopic findings. We conclude that ultrasound is a simple, accurate, inexpensive and non invasive way of diagnosing knee disorders with a learning curve.
\end{abstract}

Keywords - Arthroscopy, Diagnostic Knee tests, High Resolution Ultra sound (HRUS), Internal derangement of Knee(IDK), X-rays of Knee.

\section{INTRODUCTION}

The Difficulty in diagnosing lesions of the knee is that different lesions in the knee joint can produce similar findings. Combined lesions are more difficult to diagnose ${ }^{(1,2,3,4)}$. HRUS is non-invasive ${ }^{(5,6,7)}$, and is a safe $^{(6)}$ procedure. The sensitivity of HRUS for meniscal lesions may exceed $90 \%{ }^{(5,6,7,8,9)}$, but despite this, diagnostic arthroscopy is often advised ${ }^{(6,8,9)}$. Sensitivity and specificity are important to find out the accuracy of a modality.. The accuracy of clinical diagnosis of meniscal tears is about $75 \%$ to $80 \%$, compared with $88 \%$ to 90\% for HRUS. For lesions of articular cartilage, both the sensitivity and accuracy of HRUS are low.

Many arthroscopies which may not be required will be performed if every HRUS report is taken at face value. HRUS is a considerable advance and may well prevent patients from unnecessary operations, but it is not infallible and its technology should not dazzle clinicians. A knee, which is producing definite mechanical symptoms, will need arthroscopic surgery whatever is shown on HRUS, the latter is therefore unnecessary. If there are no mechanical symptoms HRUS may be indicated to exclude any treatable pathology.

The present comprehensive comparative study of Arthroscopy and HRUS in internal derangement of knee would guide us when to advice HRUS or proceed directly for arthroscopy.

\section{Materials and Methods}

'In this prospective study patients with symptoms due to internal derangement of knee joint were evaluated by HRUS and findings confirmed by arthroscopy at Jai Hind Centre for Ortho and Radiology, Hyderabad, India. Patients from age 16yrs to 50yrs with closed traumatic injury without fractures and arthritis were selected. HRUS and Arthroscopy was performed to all patients and documented.

We usedLinear array $17 \mathrm{MH}$ z HRUS probe ${ }^{(10)}$. Standard $30^{\circ} 4 \mathrm{~mm}$ Arthroscope with fiberoptic cold light source, gravity fed irrigation system and camera for video recording and picturising on monitor was used.

Following measures are used to evaluate a screening test. Sensitivity, Specificity, Percentage of false negatives, Percentage of false positives. The formulas are stated below.The accuracy is the percentage of patients in whom the Arthroscopy or HRUS diagnosis is correct and is calculated as follows:

$$
\text { true positives + true negatives__ X 100\% }
$$

true positives + true negatives + false positives + false negatives

Sensitivity is the ability of test to detect an abnormality. It is determined using the equation:

$$
\text { true positive results } \quad \times 100 \%
$$

true positive results + false negative results

Specificity is an assessment of the accuracy of a test result such that the more specific a test, the fewer

false positive results. It is determined using the equation :

$$
\text { True negative results } \quad \text { X } 100 \%
$$

True negative results + false positive results

\section{Observations And Results}

In the 189 patients who underwent both clinical examination and MRI, a total 69 Medial Meniscus tears, 22Lateral Meniscus tears, and 131 ACL tears, 6 PCL tears were found at Arthroscopy. 
Table 1. Reliability of HRUS as determined at Arthroscopy

\begin{tabular}{|c|c|c|c|c|}
\hline & $\begin{array}{l}\text { Medial } \\
\text { Meniscus }\end{array}$ & $\begin{array}{l}\text { Lateral } \\
\text { Meniscus }\end{array}$ & $\begin{array}{l}P C \\
L\end{array}$ & $\begin{array}{l}A C \\
L\end{array}$ \\
\hline $\begin{array}{l}\text { True } \\
\text { positive }\end{array}$ & 62 & 15 & 6 & 138 \\
\hline $\begin{array}{l}\text { True } \\
\text { negative }\end{array}$ & 111 & 164 & 183 & 10 \\
\hline $\begin{array}{l}\text { False } \\
\text { positive }\end{array}$ & 10 & 6 & 0 & 10 \\
\hline $\begin{array}{l}\text { False } \\
\text { negative }\end{array}$ & 6 & 4 & 0 & 1 \\
\hline
\end{tabular}

\section{Discussion}

We performed the present study to compare the accuracy of HRUS in the diagnosis of meniscal and ACL tears. The accuracy of HRUS reported in this study $91.33 \%$ for meniscal tears and $90.6 \%$ for ACL tears. Although proponents of HRUS believe that it can decrease the necessity for more expensive and invasive diagnostic arthroscopy and also assist in operative planning, our study shows that it does not increase the accuracy of diagnosing meniscal and ACL tears.

Zaka Khan et $\mathrm{al}^{(10)}$ felt that HRUS is a cost effective means of avoiding diagnostic arthroscopy. In our study all patients were subjected to arthroscopy to finalise HRUS diagnosis, since it is our set protocol.

Grifka et $\mathrm{al}^{(7)}$ devised a mathematical formula to assess the cost-effectiveness of HRUS. They found that if more than $78 \%$ of a patient population studied by HRUS ultimately underwent arthroscopy, it would be less expensive to bypass HRUS and proceed directly to arthroscopy.. We concur that the clinical course of the patient and not the HRUS findings alone should determine the therapeutic course.

Although HRUS has proven to be an accurate modality for diagnosing meniscal and ACL tears, it is not without its limitations. Some of the reasons for its inaccuracy in diagnosing ACL tears are readily apparent. The ACL is an intra-articular yet extra synovial structure. Therefore, in those cases where the ligament is torn but the envelope remains intact, a false negative interpretation may result. Furthermore, partial ACL tears and tears near its origin are often misinterpreted as normal. The HRUS false positive ACL diagnosis may have been secondary to hypertrophic synovial tissue through the knee, possibly obscuring precise imaging of the ACL. Mucoid or eosinophilic degeneration within the ACL can also cause a false positive HRUS reading.

Many normal anatomic structures in the knee may mimic meniscal and ligamentous pathology resulting in false positive readings. The transverse geniculate ligament may appear to be a lateral meniscal tear in the region of confluence between this ligament and its attachment to the anterior horn of the lateral meniscus. The bursa of the popliteus tendon and the ligament of Humphry may mimic a tear in the posterior horn of the lateral meniscus.

One compelling reason for prearthroscopic HRUS is preoperative planning with the thought being that a surgeon who is wary of an abnormality shown on HRUS is more likely to do extensive probing of that area and, therefore, less likely to miss the lesion. However, we feel that with thorough clinical and arthroscopic examination, the likelihood of this occurring is greatly diminished.

The weakness of this study should be pointed out. First, the study is based on decision outcome rather than patient outcome. The numbers reported are highly dependent on the skills of the surgeon and radiologists used in the study, and the use of one surgeon potentially may introduce a bias. In some cases where there were intrasubstance tear of menisci, ACL and posterior horn tear of the menisci, which were not diagnosed on arthroscopy.

\section{Conclusion}

There is no substitute for good clinical examination. Reporting of HRUS is dependent on the Radiologist and learning curve ${ }^{(11,12)}$. Do not subject all patients toHRUS, it is not the first test. HRUS should be advised to patients who have persistent pain despite conservative treatment,patients in whom two or more lesions are suspected, patients who have suspected partial meniscal tear but clinical examination is normal.

\section{REFERENCES}

[1]. H Gerngross, C. Sohn. Ultrasound Scanning For The Diagnosis Of Meniscal Lesions Of The Knee Joint.( Arthroscopy 1992 ) 8 :105110 .

[2]. J. Grifka , J. Richter , M Gumtau . Clinical And Sonographic Meniscus Diagnosis.(Orthopäde 1994) 23:102-111.

[3]. F Corbetti , G Tomasella . Meniscal Injuries Of The Knee - Arthrographic And Echographic Study.(Radiol Med 1989) 77 : 187-194.

[4]. M Fusting, HR Casser . Dynamic Examination Technique In Meniscus Sonography. (Sportverletz Sportschaden 1991) 5 : $27-36$.

[5]. HR Casser, M Fusting . Current Developments In Ultra Sonography Of The Meniscus. (Orthopäde 1993) 22: 307-316.

[6]. T Heuchemer, G Bauer, Friedrich, Bargon. Clinical Use Of Meniscus Sonography.(Bildgebung 1987-1989) 56:118-123. 
[7]. J Richter, Grifka, Fissler-Eckhoff $L$. Ultrasound Morphologic Criteria In Evaluating Meniscus Changes, An Experimental Study. ( $Z$ Orthop Ihre Grenzeb 1996 ) 134 : 137-143.

[8]. C Sohn , H Gerngross , Bahren . Meniscus Sonography - Alternative To Invasive Meniscus Diagnosis. (Dtsch Med Wochenschr 1987) $112: 581-584$.

[9]. J Mattli , P Holzach , P Soklic . Meniscus Ultrasound - A Reliable Way For The Diagnosis Of Meniscus Lesions (Unfallchir Versicherungsmed 1993) Suppl $1: 133-140$

[10]. Zaka Khan, F Zia . Ultrasound Assessment Of Internal Derangement Of The Knee. (Acta Orthop. Belg., 2006) $72-76$

[11]. HR Casser, C Sohn, Kiekenbeck . Current Evaluation Of Sonography Of The Meniscus. Results Of A Comparative Study Of Sonographic And Arthroscopic Findings. (Arch Orthop Trauma Surg 1990) 109: 150-154.

[12]. C Sohn, HR Casser, W Swobodnik. Ultrasound Criteria Of A Meniscus Lesion. (Ultraschall Med 1990) 11: 86-90. 